\title{
EQUIVALENCE OF FINITE ELEMENT METHODS FOR PROBLEMS IN ELASTICITY*
}

\author{
RICHARD S. FALK† AND MARY E. MORLEY
}

\begin{abstract}
Modifications of the Morley method for the approximation of the biharmonic equation are obtained from various finite element methods applied to the equations of linear isotropic elasticity and the stationary Stokes equations, by elimination procedures analogous to those used in the continuous case. Problems with Korn's first inequality for nonconforming $P_{1}$ elements and its implications for the approximation of the elasticity equations are also discussed.
\end{abstract}

Key words. biharmonic, stokes, elasticity, finite element

AMS(MOS) subject classifications. 65N30, 73K25

1. Introduction. It is well known that the biharmonic equation arises in several contexts in the theory of linear elasticity from the reduction of the equations of linear isotropic elasticity, in which more variables are initially present, to a single higher order equation (cf. [4]). For example, starting with the equations of plane strain isotropic elasticity

$$
\underset{\approx}{\mathbf{\sigma}}=\underset{\approx}{\epsilon}(\underset{\sim}{u}) \quad \text { in } \Omega, \quad \underset{\sim}{\operatorname{div}} \underset{\approx}{\sigma}=\underset{\sim}{f} \quad \text { in } \Omega
$$

where $\Omega$ is a simply connected bounded domain in the plane,

$$
\mathbf{A} \sigma=\frac{1+\nu}{E}[\underset{\approx}{\sigma}-\nu \operatorname{tr}(\underset{\approx}{\sigma}) \underset{\approx}{\delta}], \quad \underset{\approx}{\epsilon}(\underset{\sim}{u})=\left[\underset{\approx}{\operatorname{grad}} \underset{\sim}{u}+(\underset{\approx}{\operatorname{grad}} \underset{\sim}{u})^{t}\right] / 2
$$

$\underset{\approx}{\delta}$ is the identity, and $\operatorname{tr} \underset{\approx}{\sigma})$ denotes the trace of $\underset{\approx}{\sigma}$, and introducing the variable $p=$ $k \operatorname{tr} \underset{\approx}{\sigma})(k$ an arbitrary nonzero real number), we may easily eliminate the stresses $\underset{\approx}{\sigma}$, obtaining the equations

$$
\frac{1+\nu}{E k}(1-2 \nu) p=\operatorname{div} \underset{\sim}{u}
$$

and

$$
\frac{E}{(1+\nu)} \operatorname{div} \underset{\approx}{\epsilon}(\underset{\sim}{u})+\frac{\nu}{k} \underset{\sim}{\operatorname{grad} p}=\underset{\sim}{f}
$$

Applying the calculus identity:

$$
\underset{\sim}{\operatorname{div}}\left(\underset{\approx}{\operatorname{grad}} \underset{\sim}{u^{t}}\right)=\underset{\sim}{\operatorname{grad}}(\operatorname{div} \underset{\sim}{u})
$$

and the definition of $\underset{\approx}{\epsilon}(u),(1.2)$ may also be rewritten as

$$
\frac{E}{2(1+\nu)} \Delta \underset{\sim}{u}+\frac{1}{2 k} \underset{\sim}{\operatorname{grad}} p=\underset{\sim}{f}
$$

*Received by the editors May 15, 1989; accepted for publication January 18, 1990. This research was supported by National Science Foundation grant DMS-87-03354 (RSF).

$\dagger$ Department of Mathematics, Rutgers University New Brunswick, New Jersey 08903.

$\ddagger$ Department of Mathematics, Temple University, Philadelphia, Pennsylvania 19122. 
In the incompressible case $\left(\nu=\frac{1}{2}\right),(1.1)$ becomes div $\underset{\sim}{u}=0$ and we obtain the

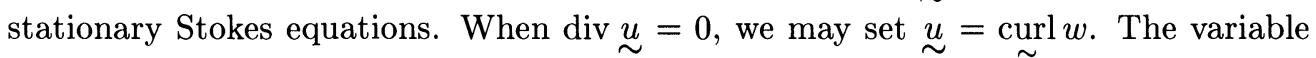
$p$ may now be eliminated by differentiating and adding the remaining equations. The resulting equation satisfied by $w$ is the biharmonic equation

$$
\mu \Delta^{2} w=g \quad \text { in } \Omega
$$

where $\mu=E /[2(1+\nu)]$ and $g=\partial f_{1} / \partial y-\partial f_{2} / \partial x$.

A second derivation, applicable when the force $\underset{\sim}{f}=\underset{\sim}{\operatorname{grad}} \phi$ for some potential $\phi$, procedes by the introduction of the Airy stress function, i.e., the stress $\underset{\approx}{\sigma}$ is written in the form

$$
\underset{\approx}{\sigma}=\left(\begin{array}{ll}
\phi & 0 \\
0 & \phi
\end{array}\right)+\left(\begin{array}{cc}
w_{y y} & -w_{x y} \\
-w_{x y} & w_{x x}
\end{array}\right)
$$

so that $\underset{\sim}{\operatorname{div}} \underset{\approx}{\sigma}=\underset{\sim}{\operatorname{grad}} \phi=\underset{\sim}{f}$. Letting $\underset{\approx}{\mathcal{A}}$ denote the Airy operator

$$
\underset{\mathcal{A}}{\approx}=\left(\begin{array}{cc}
\partial^{2} / \partial y^{2} & -\partial^{2} / \partial x \partial y \\
-\partial^{2} / \partial x \partial y & \partial^{2} / \partial x^{2}
\end{array}\right)
$$

an easy computation shows that if $\underset{\approx}{\sigma}$ is of the above form and satisfies $\underset{\approx}{\mathbf{A} \sigma}=\underset{\approx}{\epsilon}(u)$, then

$$
\frac{1+\nu}{E}\left[(1-\nu) \Delta^{2} w+(1-2 \nu) \Delta \phi\right]=\sum_{i, j=1}^{2} \mathcal{A}_{i j}(\underset{\approx}{\mathbf{A}})_{i j}=\sum_{i, j=1}^{2} \mathcal{A}_{i j} \epsilon_{i j}(\underset{\sim}{u})=0
$$

Hence, $w$ satisfies the biharmonic equation:

$$
\Delta^{2} w=-\frac{1-2 \nu}{1-\nu} \Delta \phi
$$

When appropriate boundary conditions are added to each of these equations, it is then possible to show the equivalence of various boundary value problems for the equations of elasticity, the stationary Stokes equations, and the biharmonic equation. These standard results are recalled for the reader in $\S 2$. Since this is the case, it is interesting to determine whether any finite element methods based on these formulations are also equivalent. In particular, we shall show in $\S 3$ how a modification of the Morley method for the biharmonic (cf. [6]-[9]) can be obtained from the standard continuous piecewise linear approximation of the elasticity equations in the case when $\underset{\sim}{f}=\underset{\sim}{\operatorname{grad}} \phi$, by elimination procedures analogous to those used in the continuous case. The key idea is a discrete version of the orthogonal decomposition of symmetric tensors in the form $\underset{\approx}{\epsilon} \underset{\sim}{u})+\underset{\approx}{\mathcal{A}}(w)$. In $\S 4$, we show how another modified Morley method for the biharmonic arises from the nonconforming piecewise linear approximation proposed in [3] for the stationary Stokes equations (1.3), (1.1) (with $\nu=\frac{1}{2}$ ), by writing the solution as the discrete curl of a Morley element. In a similar vein, we note that Arnold and Brezzi [1] have shown that the Hellan-Hermann-Johnson approximation of the biharmonic equation is also equivalent to a modification of the Morley method. We then compare the error estimates that can be derived for all these various versions of the Morley method. 
In $\S 5$, we consider what happens when (1.2) rather than (1.3) is used as the basis of a nonconforming $P_{1}$ finite element scheme. The analysis then depends on an appropriate discrete version of Korn's inequality for nonconforming piecewise linear elements. We show that such an inequality may not hold in certain cases, and for other cases in which it does hold, the constant may go to infinity as the mesh size approaches zero. Finally, in $\S 6$, we give a mixed formulation which is equivalent in the incompressible limit to the nonconforming $P_{1}$ approximation of the Stokes problem studied in $\S 4$ and compare it to a mixed method developed in [2] using RaviartThomas elements (cf. [10]).

2. Notation and preliminaries. We will use the usual $L^{2}$-based Sobolev spaces $H^{s}$. An undertilde to a space denotes the 2-vector-valued analogue. The undertilde is also used to denote vector-valued functions and operators, and double undertildes are used for matrix-valued objects. The letter $C$ denotes a generic constant, not necessarily the same in each occurrence. We will use various standard differential operators defined as follows:

$$
\begin{aligned}
& \underset{\sim}{\operatorname{grad}} p=\left(\begin{array}{c}
\partial p / \partial x \\
\partial p / \partial y
\end{array}\right), \quad \underset{\sim}{\operatorname{div} \tau} \underset{\approx}{\tau}=\left(\begin{array}{l}
\partial \tau_{11} / \partial x+\partial \tau_{12} / \partial y \\
\partial \tau_{21} / \partial x+\partial \tau_{22} / \partial y
\end{array}\right), \quad \underset{\sim}{\operatorname{curl} p} p=\left(\begin{array}{r}
\partial p / \partial y \\
-\partial p / \partial x
\end{array}\right), \\
& \operatorname{div} \underset{\sim}{v}=\frac{\partial v_{1}}{\partial x}+\frac{\partial v_{2}}{\partial y}, \quad \operatorname{rot} \underset{\sim}{v}=-\frac{\partial v_{1}}{\partial y}+\frac{\partial v_{2}}{\partial x} \\
& \underset{\approx}{\operatorname{grad}} \underset{\sim}{v}=\left(\begin{array}{ll}
\partial v_{1} / \partial x & \partial v_{1} / \partial y \\
\partial v_{2} / \partial x & \partial v_{2} / \partial y
\end{array}\right)
\end{aligned}
$$

and

$$
\underset{\approx}{\epsilon}(\underset{\sim}{v})=\frac{1}{2}\left[\underset{\approx}{\operatorname{grad}} \underset{\sim}{v}+(\underset{\approx}{\operatorname{grad}} \underset{\sim}{v})^{t}\right]
$$

We also define the constant tensor

$$
\underset{\approx}{\delta}=\left(\begin{array}{ll}
1 & 0 \\
0 & 1
\end{array}\right)
$$

and for any tensor $\underset{\approx}{\tau}$

$$
\operatorname{tr}(\underset{\approx}{\tau})=\underset{\approx}{\tau}: \underset{\approx}{\delta}, \quad \text { where } \underset{\approx}{\sigma}: \underset{\approx}{\tau}=\sum_{i=1}^{2} \sum_{j=1}^{2} \sigma_{i j} \tau_{i j}
$$

We now recall for the convenience of the reader some standard results on the equivalence of various boundary value problems for the equations of plane linear isotropic elasticity, the stationary Stokes equations, and the biharmonic equation. To make matters as simple as possible, while still studying the effects of different boundary conditions, we shall consider the case when the domain $\Omega$ is a convex polygon and $\Gamma_{1}$ and $\Gamma_{2}$ are disjoint open connected subsets of $\partial \Omega$ with $\bar{\Gamma}_{1} \cap \bar{\Gamma}_{2}=\partial \Omega$.

Defining

$$
\begin{aligned}
& \underset{\approx}{H^{S}}=\left\{\underset{\approx}{\tau} \in \underset{\approx}{L^{2}}(\Omega): \tau_{i j}=\tau_{j i}\right\}, \\
& \underline{\sim}^{0}(\Gamma)=\left\{\underset{\sim}{v} \in \underset{\sim}{H^{1}}(\Omega): \underset{\sim}{v}=0 \text { on } \Gamma\right\}, \\
& \underline{\sim}^{1}=\left\{\underset{\sim}{v} \in{\underset{\sim}{H}}^{1}(\Omega): \underset{\sim}{v}={\underset{\sim}{g}}_{1} \text { on } \Gamma_{1}\right\},
\end{aligned}
$$


the boundary value problem for the equations of plane strain linear isotropic elasticity may then be stated in weak form as follows.

Find $\underset{\approx}{\sigma} \underset{\approx}{H}{ }^{S}, \underset{\sim}{\in}{\underset{\sim}{\sim}}^{1}$ satisfying

$$
\begin{aligned}
& \left.\int_{\Omega} \mathbf{A} \underset{\approx}{\sigma}: \underset{\approx}{\tau} d x=\int_{\Omega} \underset{\approx}{\epsilon} \underset{\sim}{u}\right): \underset{\approx}{\tau} d x \quad \underset{\approx}{\forall} \in \underset{\approx}{H^{S}}, \\
& \int_{\Omega} \underset{\approx}{\sigma}: \underset{\approx}{\epsilon}(\underset{\sim}{\sim}) d x=-\int_{\Omega} \underset{\sim}{f} \cdot \underset{\sim}{v} d x+\int_{\Gamma_{2}} \underset{\sim}{g_{2}} \cdot \underset{\sim}{v} d s \quad \forall \underset{\sim}{\sim} \in{\underset{\sim}{\sim}}^{0}\left(\Gamma_{1}\right) .
\end{aligned}
$$

Note this corresponds to the boundary conditions $\underset{\sim}{u}=\underset{\sim}{g_{1}}$, on $\Gamma_{1}, \underset{\approx}{\sigma} \underset{\sim}{\sim}={\underset{\sim}{g}}_{2}$, on $\Gamma_{2}$. When $\mathbf{A}$ is invertible, then (2.1) implies that $\underset{\approx}{\sigma}=\mathbf{C} \underset{\approx}{\epsilon}(\underset{\sim}{u})$, where

$$
\mathbf{C} \underset{\approx}{\tau}=\mathbf{A}^{-1} \underset{\approx}{\tau}=\frac{E}{1+\nu}\left[\underset{\approx}{\tau}+\frac{\nu}{1-2 \nu} \operatorname{tr}(\underset{\approx}{\tau}) \underset{\approx}{\delta}\right]
$$

Hence, $\underset{\approx}{\sigma}$ may be eliminated and we obtain the standard displacement formulation of elasticity as follows.

Find $\underset{\sim}{u} \in{\underset{\sim}{ }}^{1}$ satisfying

$$
\int_{\Omega} \mathbf{C} \underset{\approx}{\epsilon}(\underset{\sim}{u}): \underset{\approx}{\epsilon}(\underset{\sim}{v}) d x=-\int_{\Omega} \underset{\sim}{f} \cdot \underset{\sim}{v} d x+\int_{\Gamma_{2}} \underset{\sim}{g_{2}} \cdot \underset{\sim}{v} d s \quad \forall \underset{\sim}{\forall} \in{\underset{\sim}{0}}^{0}\left(\Gamma_{1}\right)
$$

To obtain the stationary Stokes equations, set $p=k \operatorname{tr}(\underset{\approx}{\sigma})$. Choosing $\tau=q \underset{\approx}{\delta}$ in (2.1), we get that

$$
\frac{(1+\nu)}{E} \frac{1-2 \nu}{k} \int_{\Omega} p q d x=\int_{\Omega} \operatorname{div} \underset{\sim}{u} q d x \quad \forall q \in L^{2}(\Omega) .
$$

Using the definition of $\mathbf{A}$, it follows easily from (2.1) that

$$
\underset{\approx}{\sigma}=\frac{E}{(1+\nu)} \underset{\approx}{\epsilon}(u)+\frac{\nu}{k} p \underset{\approx}{\approx}
$$

Inserting this result in (2.2), we obtain

$$
\begin{array}{r}
\frac{E}{(1+\nu)} \int_{\Omega} \underset{\approx}{\epsilon}(\underset{\sim}{u}): \underset{\approx}{\epsilon}(\underset{\sim}{v}) d x+\frac{\nu}{k} \int_{\Omega} p \operatorname{div} \underset{\sim}{v} d x=-\int_{\Omega} \underset{\sim}{f} \underset{\sim}{v} d x+\int_{\Gamma_{2}} \underset{\sim}{g_{2}} \cdot \underset{\sim}{v} d s \\
\forall \underset{\sim}{v} \in{\underset{\sim}{\sim}}^{0}\left(\Gamma_{1}\right) .
\end{array}
$$

In the case when $\Gamma_{2}$ is empty so that $\underset{\sim}{v}=0$ on $\partial \Omega$, we get by twice integrating by parts and using (2.4), that

$$
\begin{aligned}
\int_{\Omega} \underset{\approx}{\epsilon}(\underset{\sim}{u}): \underset{\approx}{\epsilon}(\underset{\sim}{v}) d x & =\frac{1}{2} \int_{\Omega} \underset{\approx}{\operatorname{grad}}(\underset{\sim}{u}): \underset{\approx}{\operatorname{grad}}(\underset{\sim}{v}) d x+\frac{1}{2} \int_{\Omega} \operatorname{div} \underset{\sim}{u \operatorname{div}} \underset{\sim}{v} d x \\
& =\frac{1}{2} \int_{\Omega} \underset{\approx}{\operatorname{grad}}(\underset{\sim}{u}): \underset{\approx}{\operatorname{grad}}(\underset{\sim}{v}) d x+\frac{(1+\nu)(1-2 \nu)}{2 E k} \int_{\Omega} p \operatorname{div} \underset{\sim}{v} d x .
\end{aligned}
$$


By setting $\mu=E /[2(1+\nu)]$ and $k=-\frac{1}{2}$, and using (2.7), equation (2.6) becomes in this case

$$
\mu \int_{\Omega} \underset{\approx}{\operatorname{grad}}(\underset{\sim}{u}): \underset{\approx}{\operatorname{grad}}(\underset{\sim}{v}) d x-\int_{\Omega} p \operatorname{div} \underset{\sim}{v} d x=-\int_{\Omega} \underset{\sim}{f} \underset{\sim}{v} d x \quad \forall \underset{\sim}{v} \in \underset{\sim}{V^{0}}(\partial \Omega)
$$

and (2.4) becomes

$$
\int_{\Omega} \operatorname{div} \underset{\sim}{u} q d x=\frac{2 \nu-1}{\mu} \int_{\Omega} p q d x \quad \forall q \in L^{2}(\Omega)
$$

which, in the incompressible limit $\nu=\frac{1}{2}$, reduces to

$$
\int_{\Omega} \operatorname{div} \underset{\sim}{u} q d x=0 \quad \forall q \in L^{2}(\Omega) .
$$

Together, (2.6) and (2.10) are one form of the stationary Stokes equations. When $\Gamma_{2}$ is empty, another common formulation is (2.8) and (2.10). We note that when $-1<\nu<\frac{1}{2}, p$ may be easily eliminated from (2.8) to give another displacement formulation as follows.

Find $\underset{\sim}{\sim} \in{\underset{\sim}{ }}^{1}$ satisfying

$$
\left.\mu \int_{\Omega} \underset{\sim}{\operatorname{grad}}(\underset{\sim}{u}): \operatorname{grad}_{\approx} \underset{\sim}{v}\right) d x+\frac{\mu}{1-2 \nu} \int_{\Omega} \operatorname{div} \underset{\sim}{u} \operatorname{div} \underset{\sim}{v} d x=-\int_{\Omega} \underset{\sim}{f} \cdot \underset{\sim}{v} d x \quad \forall \underset{\sim}{v} \in{\underset{\sim}{\sim}}^{0}(\partial \Omega) .
$$

To obtain the biharmonic problem, we use (2.10) to write $\underset{\sim}{u}=\underset{\sim}{\operatorname{curl} w}$ for some $w \in W \equiv H^{2}(\Omega)$. By defining

$$
W^{0}(\Gamma)=\left\{z \in H^{2}(\Omega): z=\partial z / \partial n=0 \text { on } \Gamma\right\},
$$

and choosing $v=\underset{\sim}{\operatorname{curl}} z$ for $z \in W^{0}\left(\Gamma_{1}\right),(2.6)$ and (2.8) become

$$
\frac{E}{(1+\nu)} \int_{\Omega} \underset{\approx}{\epsilon}(\operatorname{curl} w): \underset{\sim}{\epsilon}(\operatorname{curl} z) d x=-\int_{\Omega} \underset{\sim}{f} \cdot \underset{\sim}{\operatorname{curl}} z d x+\int_{\Gamma_{2}} \underset{\sim}{g_{2}} \cdot \operatorname{curl} z d s \quad \forall z \in W^{0}\left(\Gamma_{1}\right)
$$

and

$$
\mu \int_{\Omega} \operatorname{grad}(\operatorname{curl} w): \underset{\sim}{\operatorname{grad}}(\underset{\sim}{\operatorname{curl}} z) d x=-\int_{\Omega} \underset{\sim}{f} \cdot \operatorname{curl}_{\sim} z d x \quad \forall z \in W^{0}(\partial \Omega) .
$$

These are both weak formulations of the biharmonic equation $\mu \Delta^{2} w=-\operatorname{rot} f \underset{\sim}{f}$.

We now turn to a second derivation of the biharmonic equation from the equations of elasticity, applicable when the force $\underset{\sim}{f}=\underset{\sim}{\operatorname{grad}} \phi$ for some potential $\phi$. Inserting this definition of $\underset{\sim}{f}$ in $(2.2)$ and integrating by parts, we obtain

$$
\int_{\Omega} \underset{\approx}{\sigma}: \underset{\approx}{\epsilon}(\underset{\sim}{v}) d x=\int_{\Omega} \phi \underset{\approx}{\delta}: \underset{\approx}{\epsilon}(\underset{\sim}{v}) d x+\int_{\Gamma_{2}}\left(\underset{\sim}{g_{2}}-\phi \underset{\sim}{n}\right) \cdot \underset{\sim}{v} d s \quad \forall \underset{\sim}{\forall} \in{\underset{\sim}{ }}^{0}\left(\Gamma_{1}\right)
$$

Next define a function $w^{p} \in W$ satisfying for all $\underset{\sim}{v} \in \underset{\sim}{V^{0}}\left(\Gamma_{1}\right)$

$$
\int_{\Omega} \underset{\sim}{\mathcal{A}}\left(w^{p}\right): \underset{\sim}{\epsilon}(\underset{\sim}{v}) d x=\int_{\Gamma_{2}}\left({\underset{\sim}{g}}_{2}-\phi \underset{\sim}{\sim}\right) \cdot \underset{\sim}{v} d s .
$$


Integrating by parts, we see that this is the weak form of the boundary condition

$$
\frac{\partial\left(\underset{\sim}{\left.\operatorname{curl} w^{p}\right)}\right.}{\partial s}=\underset{\sim}{g}{ }_{2}-\phi \underset{\sim}{n} \text { on } \Gamma_{2}
$$

Hence, $\underset{\approx}{\sigma}-\phi \underset{\approx}{\delta}-\underset{\approx}{\mathcal{A}}\left(w^{p}\right)$ satisfies

$$
\int_{\Omega}\left[\underset{\approx}{\sigma}-\phi \underset{\approx}{\delta}-\underset{\approx}{\mathcal{A}}\left(w^{p}\right)\right]: \underset{\approx}{\epsilon}(\underset{\sim}{v}) d x=0 \quad \forall \underset{\sim}{v} \in \underset{\sim}{V^{0}}\left(\Gamma_{1}\right)
$$

Now using the orthogonal decomposition

$$
\underset{\approx}{L^{2}}(\Omega)=\underset{\approx}{\epsilon}\left(V^{0}\left(\Gamma_{1}\right)\right)+\underset{\approx}{\mathcal{A}}\left(W^{0}\left(\Gamma_{2}\right)\right)
$$

it follows from (2.12) that

$$
\underset{\approx}{\sigma}-\phi \underset{\approx}{\delta}-\underset{\approx}{\mathcal{A}}\left(w^{p}\right)=\underset{\approx}{\mathcal{A}}\left(w^{0}\right)
$$

for some $w^{0} \in W^{0}\left(\Gamma_{2}\right)$. Inserting this result in (2.1) and choosing $\underset{\approx}{\tau}=\underset{\approx}{\mathcal{A}}(z)$ for $z \in W^{0}\left(\Gamma_{2}\right)$, we find that $w=w^{0}+w^{p}$ satisfies

$$
\int_{\Omega} \mathbf{A} \underset{\approx}{\mathcal{A}}(w): \underset{\approx}{\mathcal{A}}(z) d x=\int_{\Omega} \underset{\approx}{\epsilon}(\underset{\sim}{u}): \underset{\approx}{\mathcal{A}}(z) d x-\int_{\Omega} \mathbf{A} \phi \underset{\approx}{\delta}: \underset{\approx}{\mathcal{A}}(z) d x \quad \forall z \in W^{0}\left(\Gamma_{2}\right) .
$$

Integrating by parts and using the boundary condition, $\underset{\sim}{u}=\underset{\sim}{g}=\left(g_{11}, g_{12}\right)$ on $\Gamma_{1}$, we obtain for all $z \in W^{0}\left(\Gamma_{2}\right)$

$$
\int_{\Omega} \underset{\approx}{\mathbf{A}}(w): \underset{\approx}{\mathcal{A}}(z) d x=-\frac{(1+\nu)(1-2 \nu)}{E} \int_{\Omega} \phi \Delta z d x+\int_{\Gamma_{1}}\left(\frac{\partial g_{11}}{\partial s} \frac{\partial z}{\partial y}-\frac{\partial g_{12}}{\partial s} \frac{\partial z}{\partial x}\right) d s
$$

This is the weak form of a boundary value problem for the equation

$$
\Delta^{2} w=-\frac{1-2 \nu}{1-\nu} \Delta \phi \quad \text { in } \Omega
$$

3. From elasticity to the biharmonic via the Airy stress tensor. In this section, we consider the approximation of the equations of elasticity when $\underset{\sim}{f}=\underset{\sim}{\operatorname{grad}} \phi$, where the biharmonic equation arises through the introduction of the Airy stress tensor. To see how different types of boundary conditions are handled, we consider the boundary conditions:

$$
\underset{\sim}{\sim}{\underset{\sim}{g}}_{1} \quad \text { on } \Gamma_{1}, \quad \underset{\sim}{\sigma} \underset{\sim}{g_{2}} \quad \text { on } \Gamma_{2}
$$

We shall assume that $\Omega$ is a convex polygon and denote by $\tau_{h}$ a triangulation of $\Omega$ into triangles $T$ of diameter $\leq h$. We further assume that $\Gamma_{1}$ and $\Gamma_{2}$ are disjoint open connected subsets of $\partial \Omega$ with $\bar{\Gamma}_{1} \cup \bar{\Gamma}_{2}=\partial \Omega$, and that the two points of intersection of $\bar{\Gamma}_{1} \cap \bar{\Gamma}_{2}$ are mesh points. 
Let us now recall the standard approximation of the displacement form of the elasticity equations by continuous piecewise linear functions. By defining

$$
\begin{aligned}
& {\underset{\sim}{U}}_{h}=\left\{\underset{\sim}{v} \in\left[C^{0}(\Omega)\right]^{2}:\left.v_{i}\right|_{T} \in P_{1}(T)\right\} \\
& {\underset{\sim}{\sim}}_{h}^{0}\left(\Gamma_{1}\right)=\left\{\underset{\sim}{v} \in{\underset{\sim}{H}}_{h}: \underset{\sim}{v}=0 \text { on } \Gamma_{1}\right\}
\end{aligned}
$$

where $P_{i}(T)$ denotes the set of polynomials of degree $\leq i$ on $T$, and letting $\underset{\sim}{g}$ denote a suitable interpolant of $\underset{\sim}{g}$ into $\left.\underset{\sim}{U_{h}}\right|_{\Gamma_{1}}$, the method based on (2.3) is as follows.

Find $\underset{\sim}{u}{ }_{h} \in \underset{\sim}{U_{h}}, \underset{\sim}{u} h={\underset{\sim}{g}}_{1}^{I}$ on $\Gamma_{1}$ such that

$$
\int_{\Omega} \mathbf{C} \underset{\approx}{\epsilon}\left({\underset{\sim}{u}}_{h}\right): \underset{\approx}{\epsilon}(\underset{\sim}{v}) d x=-\int_{\Omega} \underset{\sim}{f} \cdot \underset{\sim}{v} d x+\int_{\Gamma_{2}} \underset{\sim}{g_{2}} \cdot \underset{\sim}{v} d s \quad \forall \underset{\sim}{v} \in{\underset{\sim}{U}}_{h}^{0}\left(\Gamma_{1}\right) .
$$

Once $\underset{\sim}{u_{h}}$ is computed, an approximation to the stress tensor $\underset{\approx}{\sigma}$ is given by $\underset{\approx}{\sigma_{h}}=$ $\mathrm{C} \underset{\approx}{\epsilon}(\underset{\sim}{\sim} h)$

We now show how this same approximation arises through a nonconforming approximation of the biharmonic equation using Morley elements.

First define

$$
\underset{\approx}{H_{h}}=\left\{\underset{\approx}{\tau}:\left.\tau_{i j}\right|_{T} \in P_{0}(T)\right\} \quad \text { and } \quad \underset{\approx}{H_{h}^{S}}=\left\{\underset{\approx}{\tau} \in \underset{\approx}{H_{h}}: \tau_{12}=\tau_{21}\right\}
$$

Note that $\underset{\approx}{\sigma_{h}} \in \underset{\approx}{H}$ satisfies:

$$
\begin{aligned}
& \int_{\Omega} \mathbf{A} \underset{\approx}{\sigma_{h}}: \underset{\approx}{\tau} d x=\int_{\Omega} \underset{\approx}{\epsilon}\left({\underset{\sim}{h}}_{h}\right): \underset{\approx}{\tau} d x \quad \forall \underset{\approx}{\forall} \underset{\approx}{H_{h}^{S}} \\
& \int_{\Omega} \underset{\approx}{\sigma_{h}}: \underset{\approx}{\epsilon}(\underset{\sim}{v}) d x=-\int_{\Omega} \underset{\sim}{f} \cdot \underset{\sim}{v} d x+\int_{\Gamma_{2}} \underset{\sim}{g_{2}} \cdot \underset{\sim}{v} d s \quad \forall \underset{\sim}{v} \in \underset{\sim}{U_{h}^{0}}\left(\Gamma_{1}\right) .
\end{aligned}
$$

Next observe that when $\underset{\sim}{f}=\underset{\sim}{\operatorname{grad}} \phi$, the right side may be written

$$
\int_{\Omega} \phi \underset{\approx}{\delta}: \underset{\approx}{\epsilon}(\underset{\sim}{v}) d x+\int_{\Gamma_{2}}\left(\underset{\sim}{g_{2}}-\phi \underset{\sim}{n}\right) \cdot \underset{\sim}{v} d s
$$

The key fact is now the use of a discrete version of the orthogonal decomposition (2.13). To state this discrete version, define the Morley finite element spaces

$$
\begin{aligned}
& M_{h}=\left\{w:\left.w\right|_{T} \in P_{2}(T), w\right. \text { continuous at vertices } \\
&\text { of } \left.\tau_{h}, \partial w / \partial n \text { continuous at midpoints of } \tau_{h}\right\}
\end{aligned}
$$

and

$$
\begin{aligned}
M_{h}^{0}\left(\Gamma_{2}\right)=\left\{w \in M_{h}: w=0 \text { at vertices of } \tau_{h} \cap \Gamma_{2},\right. \\
\\
\left.\partial w / \partial n=0 \text { at midpoints of edges of } \tau_{h} \cap \Gamma_{2} \cdot\right\}
\end{aligned}
$$

Let $\underset{\approx}{\mathcal{A}_{h}}(w) \in \underset{\approx}{L^{2}}(\Omega)$ be defined by $\left.\underset{\approx}{\mathcal{A}_{h}}(w)\right|_{T}=\left.\underset{\approx}{\mathcal{A}}(w)\right|_{T}$, for $w \in H^{2}(T)$. We then get the following discrete version of (2.13). 
THEOREM 3.1.

$$
\underset{\approx}{H_{h}^{S}}=\underset{\approx}{\epsilon}\left({\underset{\sim}{U}}_{h}^{0}\left(\Gamma_{1}\right)\right)+\underset{\approx}{\mathcal{A}_{h}}\left(M_{h}^{0}\left(\Gamma_{2}\right)\right)
$$

and this is an $\underset{\approx}{2}(\Omega)$ orthogonal decomposition.

Proof. Clearly

$$
\underset{\approx}{\epsilon}\left(\underset{\sim}{U_{h}^{0}}\left(\Gamma_{1}\right)\right)+\underset{\approx}{\mathcal{A}}\left(M_{h}^{0}\left(\Gamma_{2}\right)\right) \subseteq \underset{\approx}{H}{ }_{h}^{S}
$$

To show equality, we now show that the dimensions are the same. Let $v_{I}, e_{I}$, and $e_{B}$ denote the number of interior vertices, interior edges, and boundary edges, respectively, of $\tau_{h}$. Let $v_{B}^{i}$ and $e_{B}^{i}, i=1,2$ denote the number of vertices and edges, respectively, of $\tau_{h}$ on $\bar{\Gamma}_{i}$. Then $e_{B} \equiv e_{B}^{1}+e_{B}^{2}=v_{B}^{1}+v_{B}^{2}-2$, since there are two points of intersection of $\bar{\Gamma}_{1} \cap \bar{\Gamma}_{2}$. Counting degrees of freedom, we get that

$$
\begin{aligned}
& \operatorname{dim} \underset{\approx}{\mathcal{A}_{h}}\left(M_{h}^{0}\left(\Gamma_{2}\right)\right)=\operatorname{dim} M_{h}^{0}\left(\Gamma_{2}\right)=v_{I}+e_{I}+v_{B}^{1}+e_{B}^{1}-2, \\
& \operatorname{dim} \underset{\approx}{\epsilon}\left({\underset{\sim}{U}}_{h}^{0}\left(\Gamma_{1}\right)\right)=\operatorname{dim} \underset{\sim}{U_{h}^{0}}\left(\Gamma_{1}\right)=2 v_{I}+2 v_{B}^{2}-4
\end{aligned}
$$

(because of duplication of vertices). Letting $e, v$, and $T$ denote the number of edges, vertices, and triangles of $\tau_{h}$, respectively, and using the identity

$$
2 e_{I}+e_{B}=3 T\left(\Rightarrow 4 e_{I}+2 e_{B}=6 T\right)
$$

and Euler's formula

$$
T-e+v=1\left(\Rightarrow 3+3 e_{I}-3 v_{I}=3 T\right)
$$

we get

$$
\operatorname{dim} \underset{\approx}{\mathcal{A}_{h}}\left(M_{h}^{0}\left(\Gamma_{2}\right)\right)+\operatorname{dim} \underset{\approx}{\epsilon}\left({\underset{\sim}{h}}_{h}^{0}\left(\Gamma_{1}\right)\right)=3 v_{I}+e_{I}+2 e_{B}-3=3 T=\operatorname{dim} \underset{\approx}{H_{h}^{S}} .
$$

Hence, the spaces are the same.

To check the orthogonality condition, let $\underset{\sim}{v} \in \underset{\sim}{U_{h}^{0}}\left(\Gamma_{1}\right), \underset{\approx}{\mathcal{A}_{h}}(w) \in \underset{\approx}{\mathcal{A}_{h}}\left(M_{h}^{0}\left(\Gamma_{2}\right)\right)$. Then

$$
\left.\sum_{T} \int_{T} \underset{\approx}{\epsilon} \underset{\sim}{v}\right): \underset{\approx}{\mathcal{A}_{h}}(w) d x=\sum_{T} \int_{\partial T}\left(\frac{\partial v_{1}}{\partial s} \frac{\partial w}{\partial y}-\frac{\partial v_{2}}{\partial s} \frac{\partial w}{\partial x}\right) d s=0
$$

since $\partial v_{i} / \partial s$ is a constant on each edge which is continuous across neighboring triangles and $\partial w / \partial x$ and $\partial w / \partial y$ are piecewise linear functions which are continuous at the midpoints. Thus contributions from adjoining triangles cancel and there are no contributions from boundary edges because of the boundary conditions.

To use this decomposition, we first define a function $w_{h}^{p} \in M_{h}$ satisfying for all $\underset{v}{\sim} \in \underset{\sim}{U}{ }_{h}^{0}\left(\Gamma_{1}\right)$

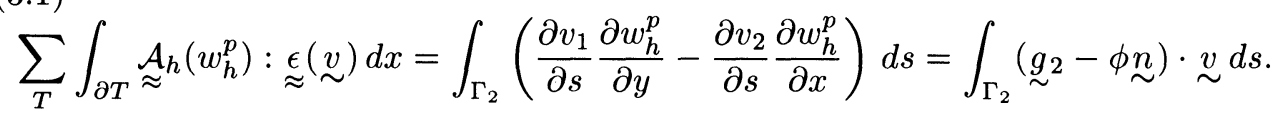

Note that this only involves assigning boundary values of $w_{h}^{p}$ and a solution may be found in the following way. Let $x_{1}$ and $x_{2}$ denote the points of intersection of $\bar{\Gamma}_{1}$ 
and $\bar{\Gamma}_{2}$. Let $w_{h}^{p}$ satisfy $\partial w_{h}^{p} / \partial n=0$ at midpoints of all interior edges of $\tau_{h}$ and of edges of $\tau_{h}$ on $\Gamma_{1}$ and $w_{h}^{p}=0$ at all interior vertices of $\tau_{h}$ and at vertices of $\tau_{h}$ on $\Gamma_{1}$, including $x_{1}$, but excluding $x_{2}$. The remaining degrees of freedom of $w_{h}^{p}$ are the values of $\partial w_{h}^{p} / \partial n$ at the midpoints of the edges of $\tau_{h}$ on $\Gamma_{2}$ and the values of $w_{h}^{p}$ at the vertices of $\tau_{h}$ on $\Gamma_{2}$, including $x_{2}$, but excluding $x_{1}$. We now show how these may be easily determined from (3.1) and the additional equations

$$
\int_{\Gamma_{2}}\left(\frac{\partial v_{1}^{p}}{\partial s} \frac{\partial w_{h}^{p}}{\partial y}-\frac{\partial v_{2}^{p}}{\partial s} \frac{\partial w_{h}^{p}}{\partial x}\right) d s=0
$$

where ${\underset{\sim}{v}}^{p} \in{\underset{\sim}{U}}^{p}=\operatorname{span}(\chi, 0),(0, \chi)$, with $\chi$ the piecewise linear function which is one at $x_{2}$ and zero at all other vertices. For $\underset{\sim}{v} \in \underset{\sim}{U_{h}^{0}}\left(\Gamma_{1}\right) \cup \underset{\sim}{U^{p}}$, it is not difficult to see that $\partial \underset{\sim}{v} / \partial s$ maps onto the space of piecewise constant vectors on $\left.\tau_{h}\right|_{\Gamma_{2}}$. Writing

$$
\int_{\Gamma_{2}}\left(\frac{\partial v_{1}^{p}}{\partial s} \frac{\partial w_{h}^{p}}{\partial y}-\frac{\partial v_{2}^{p}}{\partial s} \frac{\partial w_{h}^{p}}{\partial x}\right) d s=\int_{\Gamma_{2}}\left(\frac{\partial{\underset{v}{\sim}}^{p}}{\partial s} \cdot \underset{\sim}{\sim} \frac{\partial w_{h}^{p}}{\partial s}-\frac{\partial{\underset{\sim}{\sim}}^{p}}{\partial s} \cdot \underset{\sim}{\sim} \frac{\partial w_{h}^{p}}{\partial n}\right) d s
$$

with $\underset{n}{\sim}$ and $\underset{\sim}{\sim}$ the unit normal and tangent vectors, respectively, we get that the average values of $\partial w_{h}^{p} / \partial s$ and $\partial w_{h}^{p} / \partial n$ are uniquely determined. Since the average value of $\partial w_{h}^{p} / \partial s$ is the difference of the values of $w_{h}^{p}$ at neighboring vertices, the remaining degrees of freedom of $w_{h}^{p}$ are easily determined.

We now note that since

$$
\underset{\approx}{\sigma_{h}}-\underset{\approx}{\mathcal{A}_{h}}\left(w_{h}^{p}\right)-P_{0} \phi \underset{\approx}{\delta} \in \underset{\approx}{H}{ }_{h}^{S}
$$

we may write it in the form

$$
\underset{\approx}{\epsilon}\left(\underset{\sim}{u}{ }_{h}^{0}\right)+\underset{\approx}{\mathcal{A}_{h}}\left(w_{h}^{0}\right),
$$

where $\underset{\sim}{u}{ }_{h}^{0} \in \underset{\sim}{U_{h}^{0}}\left(\Gamma_{1}\right), w_{h}^{0} \in M_{h}^{0}\left(\Gamma_{2}\right)$. Inserting this identity into the equation:

$$
\left.\int_{\Omega} \underset{\approx}{\sigma_{h}}: \underset{\approx}{\epsilon} \underset{\sim}{v}\right) d x=\int_{\Omega} \phi \underset{\approx}{\delta}: \underset{\approx}{\epsilon}(\underset{\sim}{v}) d x+\int_{\Gamma_{2}}\left({\underset{\sim}{g}}_{2}-\phi \underset{\sim}{n}\right) \cdot \underset{\sim}{v} d s
$$

it follows immediately that

$$
\int_{\Omega}\left[\underset{\approx}{\epsilon}\left(\underset{\sim}{u}{ }_{h}^{0}\right)+\underset{\approx}{\mathcal{A}_{h}}\left(w_{h}^{0}\right)\right]: \underset{\approx}{\epsilon}(\underset{\sim}{v}) d x=0 \quad \forall \underset{\sim}{v} \in{\underset{\sim}{U}}_{h}^{0}\left(\Gamma_{1}\right)
$$

and hence by the orthogonality of the decomposition and Korn's inequality that $u_{h}^{0}=0$.

Next, setting $w_{h}=w_{h}^{0}+w_{h}^{p}$ and using the equation

$$
\int_{\Omega} \mathbf{A} \underset{\approx}{\sigma}: \underset{\approx}{\tau} d x=\int_{\Omega} \underset{\approx}{\epsilon}(\underset{\sim}{u}): \underset{\approx}{\tau} d x \quad \forall \underset{\approx}{\forall \underset{\approx}{H}} \underset{h}{S},
$$

with $\underset{\approx}{\tau}=\underset{\approx}{\mathcal{A}_{h}}(z), z \in M_{h}^{0}\left(\Gamma_{2}\right)$, we get for all $z \in M_{h}^{0}\left(\Gamma_{2}\right)$

$$
\begin{aligned}
\int_{\Omega} \underset{\approx}{\mathbf{A}} \underset{\mathcal{A}_{h}}{ }\left(w_{h}\right): \underset{\approx}{\mathcal{A}_{h}(z) d x}= & \int_{\Omega} \underset{\approx}{\epsilon}(\underset{\sim}{\sim}): \underset{\approx}{\mathcal{A}_{h}}(z)-\int_{\Omega} \mathbf{A} P_{0} \phi \underset{\approx}{\approx}: \underset{\approx}{\mathcal{A}_{h}(z) d x} \\
= & \int_{\Gamma_{1}}\left(\frac{\partial g_{11}}{\partial s} \frac{\partial z}{\partial y}-\frac{\partial g_{12}}{\partial s} \frac{\partial z}{\partial x}\right) d s \\
& -\frac{(1+\nu)(1-2 \nu)}{E} \sum_{T} \int_{T} \phi \Delta z d x
\end{aligned}
$$


where we have used integration by parts and the boundary condition $\underset{\sim}{u} h={\underset{\sim}{g}}_{1}=$ $\left(g_{11}, g_{12}\right)$. Observing that

$$
\begin{aligned}
\int_{\Omega} \mathbf{A} \underset{\approx}{\mathcal{A}_{h}}\left(w_{h}\right): & \underset{\approx}{\mathcal{A}_{h}}(z) d x \\
\quad=\frac{1+\nu}{E} & \sum_{T} \int_{T}\left(\frac{\partial^{2} w_{h}}{\partial x^{2}} \frac{\partial^{2} z}{\partial x^{2}}+2 \frac{\partial^{2} w_{h}}{\partial x \partial y} \frac{\partial^{2} z}{\partial x \partial y}+\frac{\partial^{2} w_{h}}{\partial y^{2}} \frac{\partial^{2} z}{\partial y^{2}}-\nu \Delta w_{h} \Delta z\right) d x,
\end{aligned}
$$

we have thus constructed an approximation to a weak formulation of the partial differential equation

$$
\Delta^{2} w=-\frac{1-2 \nu}{1-\nu} \Delta \phi
$$

Note that the approximation of this equation by the standard Morley method would be slightly different than the method we have derived, since the right-hand side would have the term $\sum_{T} \int_{T} \Delta \phi z d x$ instead of $\sum_{T} \int_{T} \phi \Delta z d x$, as we have obtained. It is interesting to compare the error estimates for these two methods and a third modified Morley method analysed in [1], in which the right-hand side would have the term $\sum_{T} \int_{T} \Delta \phi z_{I} d x$, where $z_{I}$ denotes the continuous piecewise linear interpolate of $z$. In order to make use of existing estimates in the literature, we shall only consider the special case when $\Gamma_{1}$ is empty, $g_{2}=0$, and $\phi=0$ on $\partial \Omega$. These conditions imply that $w$ satisfies the boundary conditions $w=\partial w / \partial n=0$.

The error estimates of interest will be those for the approximate stress. Denoting by $w_{h}^{1}, w_{h}^{2}$, and $w_{h}^{3}$ the approximate solutions of this problem produced by the standard Morley method, the Arnold-Brezzi modified Morley method, and (3.2), respectively, we define corresponding approximations $\underset{\approx}{\sigma}$ to $\underset{\approx}{\sigma}$ by $\underset{\approx}{\sigma}=P_{0} \phi \underset{\approx}{\delta}+\underset{\approx}{\mathcal{A}_{h}}\left(w_{h}^{i}\right)$. Since

$$
\left\|\underset{\approx}{\sigma}-\underset{\approx}{\sigma_{h}^{i}}\right\|_{0} \leq\left\|\phi-P_{0} \phi\right\|_{0}+\left\|w-w_{h}^{i}\right\|_{2, h}
$$

error estimates for $\underset{\approx}{\sigma}-\underset{\approx}{\sigma_{h}^{i}}$ follow directly from the results of [1] for the cases $i=1$ and $i=2$. For the case $i=3$, we use the equivalence of $\underset{\approx}{\sigma_{h}^{3}}$ and $\mathbf{C} \underset{\approx}{\epsilon}(\underset{\sim}{\sim} h)$ and standard estimates for the usual finite element approximation of the displacement form of the elasticity equations. We thus obtain the following estimates, valid on a convex polygon for $0 \leq \nu<\frac{1}{2}$.

$$
\begin{aligned}
& \|\underset{\approx}{\sigma}-\underset{\approx}{\sigma}\|_{0}^{1} \leq C h\left(\|\Delta \phi\|_{-1}+h\|\Delta \phi\|_{0}\right), \\
& \|\underset{\approx}{\sigma}-\underset{\approx}{\sigma}\|_{0}^{2} \leq C h\|\Delta \phi\|_{-1}, \\
& \|\underset{\approx}{\sigma}-\underset{\approx}{\sigma}\|_{0}^{3} \leq C h\|\underset{\sim}{\operatorname{grad}} \phi\|_{0} .
\end{aligned}
$$

Note that since $\phi=0$ on $\partial \Omega,\|\Delta \phi\|_{-1}$ and $\|\underset{\sim}{\operatorname{grad}} \phi\|_{0}$ are equivalent norms and so the last two estimates are equivalent in terms of the regularity required. Since for $\tau \in \underset{\approx}{K}=\left\{\tau: \tau_{i j} \in H^{1}(\Omega), \tau_{i j}=\tau_{j i}, \mathbf{C} \tau \underset{\approx}{\sim}=0\right.$ on $\left.\partial \Omega\right\}$

$$
\left(\underset{\approx}{\sigma}-\underset{\approx}{\sigma_{h}^{3}}, \underset{\approx}{\tau}\right)=(\mathbf{C}[\underset{\approx}{\epsilon}(\underset{\sim}{u})-\underset{\approx}{\epsilon}(\underset{\sim}{u} h)], \tau)=(\underset{\sim}{u}-\underset{\sim}{u} h, \operatorname{div}[\mathbf{C} \tau]) \leq\left\|\underset{\sim}{u}-{\underset{\sim}{u}}_{h}\right\|_{0}\left\|_{\tau}\right\|_{1}
$$

it follows directly from standard $L^{2}$ estimates for $\left\|\underset{\sim}{u}-{\underset{\sim}{\sim}}_{h}\right\|_{0}$, that

$$
\left\|\underset{\approx}{\sigma}-\sigma_{\approx}^{3}\right\|_{-1} \leq C h^{2}\|\phi\|_{1}
$$

where the negative one norm is the norm in the dual space of $\underset{\approx}{K}$. Negative norm estimates in the other two cases are not so obvious. 
4. Nonconforming $P_{1}$ and the Morley element. We next consider the relationship of some finite element approximations to the stationary Stokes and biharmonic problems

$$
\begin{aligned}
& -\mu \Delta \underset{\sim}{u}+\underset{\sim}{\operatorname{grad}} p=-f \quad \text { in } \Omega, \\
& \operatorname{div} \underset{\sim}{u}=0 \quad \text { in } \Omega, \quad \underset{\sim}{u}=0 \quad \text { on } \partial \Omega,
\end{aligned}
$$

and

$$
\mu \Delta^{2} w=g \quad \text { in } \Omega, \quad w=\partial w / \partial n=0 \quad \text { on } \partial \Omega,
$$

in the case where (4.2) is derived from (4.1) so that $g=-\operatorname{rot} \underset{\sim}{f}$.

In particular, we shall consider a method analyzed by Crouzeix and Raviart [3], in which $\underset{\sim}{u}$ is approximated by nonconforming piecewise linear functions and $p$ by piecewise constants. Again assuming that $\Omega$ is a convex polygon and $\tau_{h}$ a triangulation of $\Omega$ into triangles $T$ with diameter $\leq h$, we define

$$
\begin{aligned}
V_{h} & =\left\{\underset{\sim}{v}:\left.v_{i}\right|_{T} \in P_{1}(T), i=1,2, v_{i} \text { continuous at the midpoints of } T\right\} \\
V_{h}^{0} & =\left\{\underset{\sim}{v} \in V_{h}: \underset{\sim}{v}=0 \text { at midpoints of } T \in \partial \Omega\right\} \\
Q_{h} & =\left\{q:\left.q\right|_{T} \in P_{0}(T)\right\} .
\end{aligned}
$$

The approximation scheme is: Find $\underset{\sim}{u_{h}} \in \underset{\sim}{V_{h}^{0}}, p_{h} \in Q_{h}$ such that

$$
\begin{aligned}
& \mu \sum_{T} \int_{T} \underset{\approx}{\operatorname{grad}} \underset{\sim}{u}: \underset{\approx}{\operatorname{grad}} \underset{\sim}{v} d x-\sum_{T} \int_{T} p \operatorname{div} \underset{\sim}{\sim} d x=-\sum_{T} \int_{T} \underset{\sim}{f} \underset{\sim}{v} d x \quad \forall \underset{\sim}{v} \in V_{h}^{0}, \\
& \sum_{T} \int_{T} \operatorname{div} \underset{\sim}{u} q d x=0 \quad \forall q \in Q_{h} .
\end{aligned}
$$

Defining

$$
{\underset{\sim}{Z}}_{h}=\left\{\underset{\sim}{\sim} \in V_{h}^{0}: \sum_{T} \int_{T} \operatorname{div} \underset{\sim}{u} q d x=0 \quad \forall q \in Q_{h} .\right\}
$$

the above approximation scheme is equivalent to the following problem.

Find $\underset{\sim}{\underset{\sim}{u}} \in \underset{\sim}{Z_{h}}$ such that

$$
\mu \sum_{T} \int_{T} \operatorname{grad}_{\approx}^{u}: \operatorname{grad}_{\approx}^{\underset{\sim}{v}} d x=-\sum_{T} \int_{T} \underset{\sim}{f} \cdot \underset{\sim}{v} d x \quad \forall \underset{\sim}{v} \in Z_{h} .
$$

Now consider the Morley nonconforming finite element space $M_{h}$ defined in $\S 3$. We shall slightly simplify the notation of the previous section by defining $M_{h}^{0}=$ $M_{h}(\partial \Omega)$, i.e., $M_{h}^{0}=\left\{w \in M_{h}: w=0\right.$ at vertices of $\tau_{h} \cap \partial \Omega, \partial w / \partial n=0$ at midpoints of edges of $\left.\tau_{h} \cap \partial \Omega\right\}$.

Counting degress of freedom, it is easy to see that $\operatorname{dim} M_{h}^{0}=$ the number of interior vertices $\left(v_{I}\right)+$ the number of interior edges $\left(e_{I}\right)$. We now give a simple characterization of $Z_{h}$ using the Morley space $M_{h}^{0}$. Let $\left.\underset{\sim}{\operatorname{curl}_{h}} \psi\right|_{T}=\left.\underset{\sim}{\operatorname{curl}} \psi\right|_{T}=$ $(\partial \psi / \partial y,-\partial \psi / \partial x)_{T}$. Then we have the following theorem. 
THEOREM 4.1. $\underset{\sim}{Z}{ }_{h}=\operatorname{curl}_{h} M_{h}^{0}$.

Proof. We first show that $\operatorname{curl}_{\sim} M_{h}^{0} \subseteq \underset{\sim}{Z}$. For $\psi \in M_{h}^{0}$, let $\underset{\sim}{v}=\operatorname{curl}_{h} \psi$. Then $\underset{\sim}{\operatorname{curl}} \psi \cdot \underset{\sim}{s}=-\partial \psi / \partial n$ is continuous at midpoints of $T$ and $\operatorname{curl}_{\sim} \psi \cdot \underset{\sim}{n}=\partial \psi / \partial s$ is also, since its value along an edge of $T$ is the difference in the values of $\psi$ at the vertices determining that edge. Hence $\underset{\sim}{v} \cdot \underset{\sim}{\sim}$ and $\underset{\sim}{v} \cdot \underset{\sim}{\sim}$ and thus $\underset{\sim}{v}$ are continuous at midpoints of $\tau_{h}$. A similar argument shows that $\underset{\sim}{v}=0$ at midpoints lying on $\partial \Omega$. Thus $\underset{\sim}{v} \in \underset{\sim}{V_{h}^{0}}$. Since

$$
\sum_{T} \int_{T} \operatorname{div} \underset{\sim}{v} q d x=0 \quad \forall q \in Q_{h}
$$

$\underset{\sim}{v} \in{\underset{\sim}{h}}_{h}$. We now show equality by proving that the dimensions of the two spaces are the same. Again let $T, e$, and $v$ denote the number of triangles, edges, and vertices, respectively, in the triangulation $\tau_{h}$, and $e_{B}$ denote the number of boundary edges. First observe that $\operatorname{dim} \operatorname{curl}_{h} M_{h}^{0}=\operatorname{dim} M_{h}^{0}=e_{I}+v_{I}$. Using Euler's formula $v=e-T+1$ and the fact that $\tilde{v}-v_{I}=e_{B}$, we get that $\operatorname{dim}_{\sim} \operatorname{curl}_{h} M_{h}^{0}=2 e_{I}-T+1$. Now note that $\operatorname{dim} \underset{\sim}{Z_{h}} \geq \operatorname{dim} \underset{\sim}{V_{h}^{0}}-(T-1)=2 e_{I}-T+1$, since the divergence condition involves one constraint per triangle and at least one of these is redundant, i.e.,

$$
\sum_{T} \int_{T} \operatorname{div} \underset{\sim}{\sim} d x=\sum_{T} \int_{\partial T} \underset{\sim}{\sim} \cdot \underset{\sim}{\sim} d s=0
$$

Next observe that

$$
\operatorname{dim} \underset{\sim}{Z}{ }_{h}=\operatorname{dim} \mathcal{N}\left(\operatorname{div} \underset{\sim}{V_{h}^{0}}\right)=\operatorname{dim} \underset{\sim}{V_{h}^{0}}-\operatorname{dim} \mathcal{R}\left(\operatorname{div} \underset{\sim}{V_{h}^{0}}\right)=2 e_{I}-\operatorname{dim} \mathcal{R}\left(\operatorname{div} \underset{\sim}{V_{h}^{0}}\right)
$$

where $\mathcal{N}$ and $\mathcal{R}$ denote the null space and range respectively. Hence, to complete the proof, we need only show that $\operatorname{dim} \mathcal{R}\left(\operatorname{div} \underset{\sim}{V_{h}^{0}}\right) \geq T-1$. This is easily done by induction on $T$. When $T=2$, let $\underset{\sim}{u}$ be zero at the midpoints of boundary edges and $\underset{u}{\sim}$ be one of the unit normal vectors at the midpoint of the common edge. A simple computation shows that $\operatorname{div} \underset{\sim}{u} \neq 0$. Assuming the result is true for some $T_{1} \geq 2$, add another triangle in such a way that the number of interior edges increases by at least one. Now take $\underset{\sim}{u}=0$ at all midpoints except the midpoint of the new interior edge, where we again take it to be one of the unit normals to this edge. Again $\operatorname{div} \underset{\sim}{u} \neq 0$ and div $\underset{\sim}{u}$ is not contained in the range of the divergence operator on $T_{1}$ triangles. Hence the range of div has increased by at least one, and the result now follows by induction.

Using this result, we may substitute $\underset{\sim}{u}=\operatorname{curl} w, \underset{\sim}{v}=\underset{\sim}{\operatorname{curl}} z$ in the equation

$$
\mu \sum_{T} \int_{T} \underset{\approx}{\operatorname{grad}} \underset{\sim}{u}: \underset{\approx}{\operatorname{grad}} \underset{\sim}{v} d x=-\sum_{T} \int_{T} \underset{\sim}{f} \underset{\sim}{v} d x \quad \forall \underset{\sim}{v} \in \underset{\sim}{Z} h
$$

thus obtaining

$$
\mu \sum_{T} \int_{T}\left(\frac{\partial^{2} w}{\partial x^{2}} \frac{\partial^{2} z}{\partial x^{2}}+2 \frac{\partial^{2} w}{\partial x \partial y} \frac{\partial^{2} z}{\partial x \partial y}+\frac{\partial^{2} w}{\partial y^{2}} \frac{\partial^{2} z}{\partial y^{2}}\right) d x=\sum_{T} \int_{T}\left(-f_{1} \frac{\partial z}{\partial y}+f_{2} \frac{\partial z}{\partial x}\right) d x
$$


Note that this is a slight modification of the usual Morley method for the biharmonic problem. In the usual Morley method, the right-hand side of (4.3) is replaced by

$$
\sum_{T} \int_{T}\left(\frac{\partial f_{1}}{\partial y}-\frac{\partial f_{2}}{\partial x}\right) z d x
$$

For $z \in M_{h}^{0}$, these are not the same.

Once again, it is interesting to compare the error estimates for these two methods and the modified Morley method analysed in [1], in which the function $z$ in (4.4) is replaced by its continuous piecewise linear interpolate. Denote by $w_{h}^{1}, w_{h}^{2}$, and $w_{h}^{3}$, the approximate solutions produced by the standard Morley method, the Arnold-Brezzi modified Morley method, and (4.3), respectively. Then we have for the cases $i=1,2$ from [1] and for $i=3$ from [3] and the fact that $\underset{\sim}{u}=\underset{\sim}{\operatorname{curl}_{h}} w_{h}$, the following error estimates valid on a convex polygon.

$$
\begin{aligned}
& \left\|w-w_{h}^{1}\right\|_{2, h} \leq C h\left(\|\operatorname{rot} \underset{\sim}{f}\|_{-1}+h\|\operatorname{rot} \underset{\sim}{f}\|_{0}\right), \\
& \left\|w-w_{h}^{2}\right\|_{2, h} \leq C h\|\operatorname{rot} \underset{\sim}{f}\|_{-1}, \\
& \left\|w-w_{h}^{3}\right\|_{2, h} \leq C h\|\underset{\sim}{f}\|_{0}, \\
& \left\|w-w_{h}^{1}\right\|_{1, h} \leq C h^{2}\left(\|\operatorname{rot} \underset{\sim}{f}\|_{-1}+\|\operatorname{rot} \underset{\sim}{f}\|_{0}\right), \\
& \left\|w-w_{h}^{2}\right\|_{1, h} \leq C h^{2}\left(\|\operatorname{rot} \underset{\sim}{f}\|_{-1}+\|\operatorname{rot} \underset{\sim}{f}\|_{0}\right), \\
& \left\|w-w_{h}^{3}\right\|_{1, h} \leq C h^{2}\|f\|_{0},
\end{aligned}
$$

where

$$
\begin{aligned}
\|w\|_{2, h}^{2} & =\sum_{T} \int_{T}\left[\left(\frac{\partial^{2} w}{\partial x^{2}}\right)^{2}+2\left(\frac{\partial^{2} w}{\partial x \partial y}\right)^{2}+\left(\frac{\partial^{2} w}{\partial y^{2}}\right)^{2}\right] d x, \\
\|w\|_{1, h}^{2} & =\sum_{T} \int_{T} \mid \underset{\sim}{\left.\operatorname{grad} w\right|^{2} d x .}
\end{aligned}
$$

To compare these, we note that by the Helmholtz decomposition, $f$ may be written in the form $\underset{\sim}{f}=\underset{\sim}{\operatorname{curl}} q+\underset{\sim}{\operatorname{grad}} r$, where $q \in H_{0}^{1}(\Omega)$ and $r \in H^{1}(\Omega)$, and that this is an orthogonal decomposition in $\underset{\sim}{L^{2}}(\Omega)$. Hence,

$$
\|\operatorname{rot} \underset{\sim}{f}\|_{-1}=\|\Delta q\|_{-1}=\|\underset{\sim}{\operatorname{curl}} q\|_{0},
$$

while

$$
\|\underset{\sim}{f}\|_{0}^{2}=\|\underset{\sim}{\operatorname{curl}} q\|_{0}^{2}+\|\underset{\sim}{\operatorname{grad}} r\|_{0}^{2} .
$$

If we are given the function $\underset{\sim}{f}$, then the $\|\cdot\|_{2, h}$ estimate for $w_{h}^{2}$ is somewhat better than that for $w_{h}^{3}$, and definitely better than the corresponding estimate for $w_{h}^{1}$. In the $\|\cdot\|_{1, h}$, however, the estimate for $w_{h}^{3}$ is the best in terms of regularity required for the data. If we think of the function $g$ as given, then by $\operatorname{setting} f=\operatorname{curl} q$, where $q \in H_{0}^{1}$ satisfies $\Delta q=g$ in $\Omega$, we may replace $\|f\|_{0}$ by $\|g\|_{-1}$ in the error estimates for $w_{h}^{3}$. This would make the estimates for $w_{h}^{3}$ the best in both cases. The problem with this, of course, is that it requires the exact solution of Poisson's equation. In fact, it is not difficult to show that if $\mathrm{q}$ is approximated by its Ritz projection into continuous piecewise polynomials of degree $\geq 2$, then the same estimates hold. This extra work may only be worthwhile if $\underset{\sim}{f} \in \underset{\sim}{L^{2}}(\Omega)$, but $\operatorname{rot} \underset{\sim}{f} \notin L^{2}(\Omega)$. 
5. Nonconforming $P_{1}$ approximation of the equations of elasticity. In the previous section, we considered the approximation of the Stokes problem by nonconforming $P_{1}$ elements. If we view this equation as having arisen as the incompressible limit of the equations of elasticity, then it is important to note that the form of the Stokes equations we considered in $\S 4$ was dependent on the choice of pure displacement boundary conditions. If we had considered pure traction or mixed boundary conditions instead, the the natural bilinear form would have been

$$
\int_{\Omega} \mathbf{C} \underset{\approx}{\epsilon}(\underset{\sim}{u}): \underset{\approx}{\epsilon}(\underset{\sim}{\sim}) d x \text { instead of } \int_{\Omega} \underset{\approx}{\operatorname{grad}} \underset{\sim}{u}: \underset{\approx}{\operatorname{grad}} \underset{\sim}{v} d x
$$

Although this distinction is not crucial for conforming finite element methods, it is crucial for nonconforming $P_{1}$ elements. In this section, we show why this is so by considering the nonconforming piecewise linear approximation of the equations of linear isotropic elasticity, subject to the boundary conditions $\underset{\sim}{u}=0$ on $\Gamma_{1}, \underset{\approx}{\sigma} \underset{\sim}{\sim}=\underset{\sim}{g}$ on $\Gamma_{2}$. A natural method is as follows.

Find $\underset{\approx}{\sigma_{h}} \in \underset{\approx}{H_{h}^{S}}, \underset{\sim}{u}{ }_{h} \in \underset{\sim}{V_{h}^{0}}$ such that

$$
\begin{aligned}
& \left.\int_{\Omega} \mathbf{A} \underset{\approx}{\sigma_{h}}: \underset{\approx}{\tau} d x=\sum_{T} \int_{T} \underset{\approx}{\epsilon} \underset{\sim}{u_{h}}\right): \underset{\approx}{\tau} d x \quad \forall \underset{\approx}{\underset{\sim}{\tau} \underset{\approx}{H_{h}^{S}}} \\
& \left.\sum_{T} \int_{T} \underset{\approx}{\sigma_{h}}: \underset{\approx}{\epsilon} \underset{\sim}{v}\right) d x=-\int_{\Omega} \underset{\sim}{f} \cdot \underset{\sim}{v} d x+\int_{\Gamma_{2}} \underset{\sim}{g} \cdot \underset{\sim}{v} d s \quad \forall \underset{\sim}{v} \in{\underset{\sim}{\sim}}_{h}^{0},
\end{aligned}
$$

where now $V_{h}^{0}=\left\{\underset{\sim}{v} \in V_{h}: \underset{\sim}{v}=0\right.$ at midpoints of $T$ on $\left.\Gamma_{1}\right\}$. When $\mathbf{A}$ is invertible, this system is easily seen to be equivalent to the pure displacement method as follows.

Find $\underset{\sim}{u}{ }_{h} \in{\underset{\sim}{\sim}}_{h}^{0}$ such that

$$
\sum \int_{T} \mathbf{C} \underset{\approx}{\epsilon}(\underset{\sim}{u}): \underset{\approx}{\epsilon}(\underset{\sim}{v}) d x=-\int_{\Omega} \underset{\sim}{f} \cdot \underset{\sim}{v} d x+\int_{\Gamma_{2}} \underset{\sim}{g} \cdot \underset{\sim}{v} d s \quad \forall \underset{\sim}{v} \in{\underset{\sim}{h}}_{h}^{0}
$$

When $\nu=\frac{1}{2}$, it is also equivalent to the following approximation of the Stokes equations.

Find $\underset{\sim}{u} \in{\underset{\sim}{V}}_{h}^{0}, p_{h} \in Q_{h}$ such that for all $\underset{\sim}{v} \in \underset{\sim}{V_{h}^{0}}$ and $q \in Q_{h}$

$$
\begin{aligned}
& 2 \mu \sum_{T} \int_{T} \mathbf{C} \underset{\sim}{\epsilon}(\underset{\sim}{u}): \underset{\sim}{\epsilon}(\underset{\sim}{v}) d x-\sum_{T} \int_{T} p \operatorname{div} \underset{\sim}{\sim} d x=-\sum_{T} \int_{T} \underset{\sim}{f} \cdot \underset{\sim}{v} d x+\int_{\Gamma_{2}} \underset{\sim}{g} \cdot \underset{\sim}{v} d s, \\
& \sum_{T} \int_{T} \operatorname{div} \underset{\sim}{u} q d x=0 .
\end{aligned}
$$

The key step in the analysis of any of these equivalent formulations is a proof of a discrete version of Korn's inequality, i.e.,

$$
\left.\sum_{T}\|\underset{\approx}{\operatorname{grad}} \underset{\sim}{u}\|_{0, T}^{2} \leq K \sum_{T} \| \underset{\approx}{\epsilon} \underset{\sim}{u}\right) \|_{0, T}^{2}
$$

Unfortunately, this inequality does not always hold. In particular, consider the case when $\Gamma_{1}$ is empty so that no boundary conditions are imposed on $\underset{\sim}{u}$. On the continuous 
level, Korn's inequality holds if we factor out the three-dimensional space $(a+b y, c-$ $b x)$ of rigid motions. On the discrete level, the dimension of $\left\{\underset{\sim}{u} \in \underset{\sim}{V_{h}}: \underset{\sim}{\epsilon}(\underset{\sim}{u})=\right.$ 0 on each $T\}$ is greater than or equal to $2 e-3 T \geq e_{B}$, where $e, T$, and $e_{B}$ are defined as in the previous sections. Hence, removing global rigid motions is not enough for the inequality to be satisfied. A more thorough discussion of Korn's inequality in this case for nonconforming piecewise linear, quadratic, and cubic finite elements may be found in [5].

If we consider the case when $\underset{\sim}{u}=0$ on the boundary, then the situation is more complicated. The following example shows that for some meshes, Korn's inequality fails. Consider the triangulation in Fig. 1.

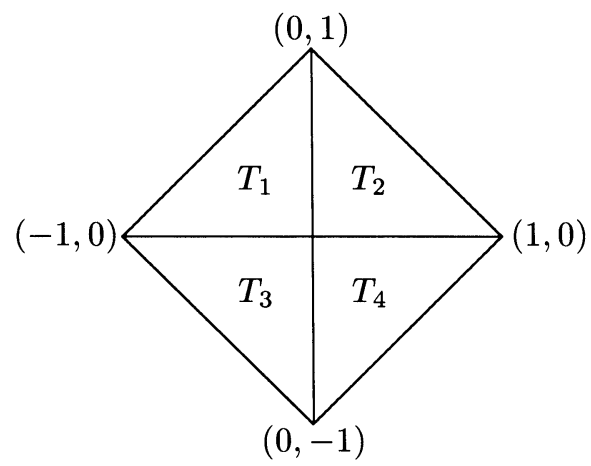

FIG. 1

Then it is easy to check that $\underset{\sim}{u}$ of the form

$$
\begin{aligned}
& \underset{\sim}{\sim}=\left(\begin{array}{l}
1 / 2-y \\
1 / 2+x
\end{array}\right) \quad \text { in } T_{1}, \quad \underset{\sim}{\sim}=\left(\begin{array}{l}
y-1 / 2 \\
1 / 2-x
\end{array}\right) \text { in } T_{2}, \\
& \stackrel{u}{\sim}=\left(\begin{array}{c}
1 / 2+y \\
-1 / 2-x
\end{array}\right) \quad \text { in } T_{3}, \quad \underset{\sim}{\sim}=\left(\begin{array}{l}
-1 / 2-y \\
-1 / 2+x
\end{array}\right) \text { in } T_{4}
\end{aligned}
$$

satisfies $\underset{\sim}{u} \in V_{h}^{0}, \underset{\approx}{\operatorname{grad}} \underset{\sim}{u} \neq 0$, but $\underset{\approx}{\epsilon} h(\underset{\sim}{u})=0$. Hence, Korn's inequality fails.

A second possibility is that Korn's inequality may hold, but the constant will approach infinity as $h$ approaches zero. To see an example of this, consider the case of a uniform mesh of isosceles right triangles of minimum side $h$ defined on $\Omega=(0,1) \times(0,1)$ (see Fig. 2).

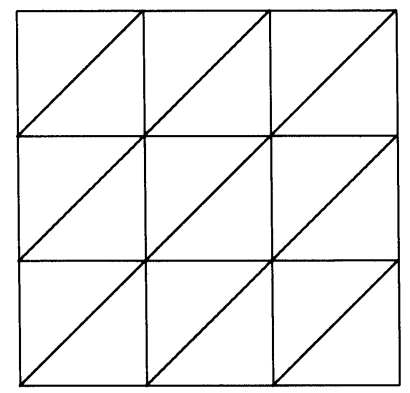

FIG. 2 
For this mesh, we shall first prove the following theorem.

THEOREM 5.1. If $\underset{\sim}{u} \in V_{h}^{0}$ satisfies $\underset{\approx}{\epsilon}(\underset{\sim}{\sim})=0$, then $\underset{\sim}{u}=0$.

Proof. The proof follows inductively from the following two lemmas, starting from the triangle in the upper left-hand corner of the square.

LEMMA 5.2. If $\underset{\sim}{\epsilon}(\underset{\sim}{u})=0$ in a triangle $T$ and $\underset{\sim}{u}$ vanishes at the midpoints of two edges of $T$, then $\underset{\sim}{\sim}=0$ in $T$.

Proof. Since $\underset{\sim}{\epsilon}(u)=0, \underset{\sim}{u}$ is a rigid motion, i.e., it has the form

$$
\stackrel{u}{\sim}=\left(\begin{array}{l}
a+b y \\
c-b x
\end{array}\right)
$$

A simple calculation shows that if $\underset{\sim}{u}$ vanishes at two distinct points, it must be identically zero.

Lemma 5.3. Let $T_{1}$ and $T_{2}$ be two triangles with a common edge e. Let $P_{3}$ denote the midpoint of $e$ and $P_{1}=\left(x_{1}, y_{1}\right)$ and $P_{2}=\left(x_{2}, y_{2}\right)$ denote the midpoints of a noncommon edge of $T_{1}$ and $T_{2}$, respectively. Suppose $\underset{\sim}{\sim}$ is a function defined on $T_{1} \cup T_{2}$ that is continuous at $P_{3}$, vanishes at $P_{1}$ and $P_{2}$, and satisfies $\underset{\sim}{\epsilon}(\underset{\sim}{\sim})=0$ in $T_{1} \cup T_{2}$. If the points $P_{1}, P_{2}, P_{3}$ do not lie on a line, then $\underset{\sim}{u}=0$ in $T_{1} \cup T_{2}$.

Proof. To simplify the computation, we may take (without loss of generality) the midpoint $P_{3}$ of $e$ to be $(0,0)$. First note that the equation of the line through the points $P_{3}$ and $P_{1}$ is given by $-y_{1} x+x_{1} y=0$. Since the point $P_{2}$ is not on this line, $-x_{1} y_{2}+x_{2} y_{1} \neq 0$.

Now since $\underset{\sim}{\epsilon} \underset{\sim}{u})=0$ on each triangle, $\underset{\sim}{u}$ will have the form

$$
\underset{\sim}{u}=\left(\begin{array}{l}
a+b y \\
c-b x
\end{array}\right) \quad \text { in } T_{1}, \quad \underset{\sim}{u}=\left(\begin{array}{l}
a+d y \\
c-d x
\end{array}\right) \quad \text { in } T_{2} .
$$

The constants $a$ and $c$ are the same on the two triangles, since $\underset{\sim}{u}$ is continuous at $P_{3}$. Since $\underset{\sim}{u}=0$ at $P_{1}$ and $P_{2}$

$$
a+b y_{1}=0, \quad c-b x_{1}=0, \quad a+d y_{2}=0, \quad c-d x_{2}=0 .
$$

Hence,

$$
b x_{1}-d x_{2}=0, \quad b y_{1}-d y_{2}=0 .
$$

Since it was shown above that the determinant $-x_{1} y_{2}+x_{2} y_{1} \neq 0, b=d=0$. This implies that $a=c=0$ and so $\underset{\sim}{u}=0$ in $T_{1} \cup T_{2}$.

From Theorem 5.1, it follows that $\|\underset{\sim}{\epsilon}(\underset{\sim}{u})\|_{0}$ is a norm on $\underset{\sim}{V_{h}^{0}}$. Since all norms are equivalent on the finite-dimensional space $\underset{\sim}{V_{h}^{0}},(5.1)$ holds for the uniform mesh under consideration for some constant $\mathrm{K}$. We now show that for this uniform mesh, the constant $K$ is at least $O\left(h^{-1 / 2}\right)$ and hence tends to infinity as $h$ tends to zero. Setting

$$
x_{i}=(i+1 / 2) h, \quad y_{j}=(j+1 / 2) h, \quad i, j=0,1, \cdots, N-1,
$$

we define

$$
\stackrel{u}{\sim}=(-1)^{i+j+1}\left(\begin{array}{c}
y-y_{j} \\
x_{i}-x
\end{array}\right)
$$


for $i h \leq x \leq(i+1) h, j h \leq y \leq(j+1) h, i, j=1,2, \cdots, N-2$ and $\underset{\sim}{u}=0$ at all remaining degrees of freedom.

Then, for example, $\underset{\sim}{u}=0$ on the triangle with vertices $(i h, 0),([i+1] h, 0),(i h, h)$, and $\underset{\sim}{u}=(-1)^{i+1}\left(\begin{array}{c}y-h / 2 \\ 0\end{array}\right)$ on the triangle with vertices $([i+1] h, 0),(i h, h),([i+1] h, h)$. An explicit computation shows that

$$
\|\underset{\approx}{\epsilon}(\underset{\sim}{u})\|_{0}^{2}=4 \sum_{i=1}^{N-2} \int_{T} 2 d x=4 h^{2}(N-2)=4 h(1-2 h)
$$

and

$\|\underset{\approx}{\operatorname{grad}} h \underset{\sim}{u}\|_{0}^{2}=4 \sum_{i=1}^{N-2} \int_{T} 1 d x+2(1-2 h)^{2}=2 h^{2}(N-2)+2(1-2 h)^{2}=2(1-2 h)(1-h)$.

Hence,

$$
\frac{\left\|\underset{\approx}{\operatorname{grad}_{h}} \underset{\sim}{u}\right\|_{0}}{\|\underset{\sim}{\epsilon} h(\underset{\sim}{u})\|_{0}}=\left(\frac{1-h}{2 h}\right)^{1 / 2}=O\left(h^{-1 / 2}\right)
$$

6. A nonconforming mixed approximation of the elasticity equations. In the case of pure displacement boundary conditions, we may easily avoid the difficulty of the last section by basing our nonconforming $P_{1}$ finite element approximation of the elasticity equations on the weak formulations (2.8) or (2.11) rather than (2.3) or (2.6). The method based on (2.8) would be as follows.

Find $\underset{\sim}{u_{h}} \in \underset{\sim}{V_{h}^{0}}, p_{h} \in Q_{h}$ such that

$$
\begin{gathered}
\mu \sum_{T} \int_{T} \underset{\approx}{\operatorname{grad}} \underset{\sim}{u_{h}}: \underset{\approx}{\operatorname{grad}} \underset{\sim}{\underset{\sim}{v}} d x-\sum_{T} \int_{T} p_{h} \operatorname{div} \underset{\sim}{\underset{\sim}{v}} d x=-\int_{\Omega} \underset{\sim}{f} \cdot \underset{\sim}{v} d x \quad \forall \underset{\sim}{v} \in \underset{\sim}{V_{h}^{0}} \\
\sum_{T} \int_{T} \operatorname{div} \underset{\sim}{u} q d x=\frac{2 \nu-1}{\mu} \int_{\Omega} p_{h} q d x \quad \forall q \in Q_{h}
\end{gathered}
$$

We note that when $-1<\nu<\frac{1}{2}, p_{h}$ may be easily eliminated as in the continuous case to give the pure displacement approximation of (2.11).

Find $\underset{\sim}{u_{h}} \in \underset{\sim}{V_{h}^{0}}$ such that

$\mu \sum_{T} \int_{T} \underset{\approx}{\operatorname{grad}} \underset{\sim}{u_{h}}: \underset{\sim}{\operatorname{grad}} \underset{\sim}{v} d x+\frac{\mu}{1-2 \nu} \sum_{T} \int_{T} \operatorname{div} \underset{\sim}{u_{h}} \operatorname{div} \underset{\sim}{v} d x=-\int_{\Omega} \underset{\sim}{f} \underset{\sim}{v} d x \underset{\sim}{\forall} \in \underset{\sim}{\sim}$.

The incompressible limit of the first of these approximations is the approximation considered in $\S 4$. This approximation is also equivalent to a mixed finite element approximation of the elasticity equations, which is similar to the one developed in [2] for the purpose of alleviating the requirement of symmetric stress tensors. We end the paper by deriving this equivalent mixed formulation beginning from (6.1), (6.2) and comparing it to the method of [2].

Define a variable

$$
\underset{\approx}{\rho_{h}}=\mu \underset{\approx}{\operatorname{grad}} \underset{\sim}{u}-p_{h} \underset{\approx}{\delta}
$$


Taking the trace of this equation, we get that $\left.\operatorname{tr} \underset{\sim}{\rho_{h}}\right)=\mu \operatorname{div} \underset{\sim}{u_{h}}-2 p_{h}$. Since (6.2) implies that on each triangle $T, \operatorname{div} \underset{\sim}{u_{h}}=[(2 \nu-1) / \mu] p_{h}$, we obtain $\operatorname{tr}\left({\underset{\approx}{\rho}}_{h}\right)=(2 \nu-3) p_{h}$. Using this result and (6.4), we find that $\underset{\sim}{\operatorname{grad}} \underset{\sim}{u}=\mathbf{B} \underset{\sim}{\rho_{h}}$, where

$$
\mathbf{B} \tau=\frac{1}{\mu}\left[\underset{\approx}{\approx}-\frac{1}{3-2 \nu} \operatorname{tr}(\underset{\approx}{\tau}) \underset{\approx}{\delta}\right]
$$

Taking the weak form of this equation, and inserting (6.4) in (6.1), we see that $\rho_{\sigma} h$, ${\underset{u}{h}}_{h}$ satisfies the following mixed formulation.

Find $\underset{\approx}{\rho_{h}} \in \underset{\approx}{H_{h}}, \underset{\sim}{u_{h}} \in \underset{\sim}{V_{h}^{0}}$ such that

$$
\begin{array}{ll}
\int_{\Omega} \mathbf{B} \underset{\approx}{\rho_{h}}: \underset{\approx}{\tau} d x=\sum_{T} \int_{T} \underset{\approx}{\operatorname{grad}} \underset{\sim}{u}: \underset{\approx}{\tau} d x & \forall \underset{\approx}{\sim} \in \underset{\approx}{H_{h}}, \\
\sum_{T} \int_{T} \underset{\approx}{\rho_{h}}: \underset{\approx}{\operatorname{grad}} \underset{\sim}{v} d x=-\int_{\Omega} \underset{\sim}{f} \cdot \underset{\sim}{v} d x & \forall \underset{\sim}{v} \in{\underset{\sim}{V_{h}^{0}} .}^{\sim}
\end{array}
$$

To obtain (6.1), (6.2) from the mixed formulation (6.5), (6.6), we define $p_{h} \in Q_{h}$ by $p_{h}=\operatorname{tr}\left(\varrho_{h}\right) /(2 \nu-3)$. Then on every triangle $T,(6.5)$ implies that $\underset{\approx}{\operatorname{grad}} \underset{\sim}{\sim}{ }_{h}=$ $\mathbf{B}{\underset{\approx}{\rho} h}=\left[\rho_{\approx} h+p_{h} \delta\right] / \mu$, so

$$
\underset{\approx}{\rho_{h}}=\mu \underset{\approx}{\operatorname{grad}} \underset{\sim}{u}-p_{h} \underset{\approx}{\delta}
$$

Inserting this result in (6.6), we obtain (6.1). Now taking the trace of (6.7) and using the definition of $p_{h}$, we find that on each triangle $T$, div $\underset{\sim}{u}=[(2 \nu-1) / \mu] p_{h}$. This equation is equivalent to (6.2).

Although $\underset{\approx}{\sigma}$ is not a variable in any of these formulations, an approximation to $\underset{\approx}{\sigma}$ may be easily recovered based on the formula (2.5), i.e.,

$$
\underset{\approx}{\sigma_{h}}=2 \mu \underset{\approx}{\epsilon} h(\underset{\sim}{u} h)-2 \nu p_{h} \underset{\approx}{\delta}
$$

It is easy to check that this is equivalent to obtaining $\underset{\approx}{\sigma_{h}}$ from ${\underset{\approx}{ }}_{h}$ by the formula

$$
\left.\underset{\approx}{\sigma_{h}}=\underset{\approx}{\rho_{h}}+\underset{\approx}{\rho} \rho^{t}+\frac{2(1-\nu)}{2 \nu-3} \operatorname{tr} \underset{\approx}{\rho_{h}}\right) \underset{\approx}{\delta}
$$

We now compare (6.5), (6.6) to the method of [2] in the special case where the lowest order Raviart-Thomas-Nedelec elements are used. To do so, we define the lowest order Raviart-Thomas spaces

$$
\begin{aligned}
& R T_{0}(T)=\{\underset{\sim}{q}=(a+b x, c+b y): a, b, c \in \mathbb{R}\}, \\
& R T_{0}^{-1}\left(\tau_{h}\right)=\left\{\underset{\sim}{q} \in\left[L^{2}(\Omega)\right]^{2}:\left.\underset{\sim}{q}\right|_{T} \in R T_{0}(T), \forall T \in \tau_{h}\right\}, \\
& R T_{0}^{0}\left(\tau_{h}\right)=\left\{\underset{\sim}{q} \in R T_{0}^{-1}\left(\tau_{h}\right): \underset{\sim}{q} \cdot \underset{\sim}{n} \text { continuous across interelement boundaries }\right\} \\
& M_{0}^{-1}\left(\tau_{h}\right)=\left\{v \in L^{2}(\Omega):\left.v\right|_{T} \in P_{0}(T), \forall T \in \tau_{h}\right\} .
\end{aligned}
$$


We then define $\underset{\approx}{R}=R T_{0}^{0}\left(\tau_{h}\right) \times R T_{0}^{0}\left(\tau_{h}\right), \underset{\approx}{R_{h}^{-1}}=R T_{0}^{-1}\left(\tau_{h}\right) \times R T_{0}^{-1}\left(\tau_{h}\right)$, and $\underset{\sim}{S}{ }_{h}=$ $M_{0}^{-1}\left(\tau_{h}\right) \times M_{0}^{-1}\left(\tau_{h}\right)$. The method of [2] using these spaces may then be stated as follows.

Find $\underset{\approx}{\rho_{h}^{m}} \in \underset{\approx}{R}, \underset{\sim}{u}{ }_{h}^{m} \in \underset{\sim}{S}$ satisfying

$$
\begin{gathered}
\int_{\Omega} \mathbf{B} \underset{\approx}{\rho_{h}^{m}}: \underset{\approx}{\tau} d x+\int_{\Omega} \underset{\sim}{u}{ }_{h}^{m} \underset{\sim}{\operatorname{div}} \underset{\approx}{\tau} d x=0 \quad \forall \underset{\approx}{\forall} \in \underset{\approx}{R_{h}^{0}}, \\
\int_{\Omega} \underset{\sim}{\operatorname{div}} \underset{\approx}{\rho_{h}^{m}} \cdot \underset{\sim}{v} d x=\int_{\Omega} \underset{\sim}{f} \underset{\sim}{v} d x \quad \forall \underset{\sim}{\forall} \in \underset{\sim}{S} .
\end{gathered}
$$

To show the relationship to (6.5), (6.6), we use the ideas of [1] for the secondorder scalar problem to give an equivalent formulation of (6.8), (6.9). Following [1], we introduce piecewise constant Lagrange multipliers $\underset{\sim}{\lambda_{h}}$ to eliminate the interelement continuity on $\underset{\approx}{R_{h}^{0}}$ and then define a function $\underset{\sim}{\psi_{h}} \in \underset{\sim}{V_{h}^{0}} \cup \underset{\sim}{B^{3}}\left(\tau_{h}\right)$ satisfying

$$
\int_{e}\left({\underset{\sim}{\sim}}_{h}-{\underset{\sim}{\lambda}}_{h}\right) d s=0, \quad \int_{\Omega}\left({\underset{\sim}{\sim}}_{h}-{\underset{\sim}{u}}_{h}^{m}\right) d x=0
$$

for each edge $e$ and every triangle $T$ of $\tau_{h}$, where $\underset{\sim}{B^{3}}\left(\tau_{h}\right)$ denotes the space of cubic bubble functions. Inserting these definitions and integrating by parts, we find that $\underset{\sim}{\rho} \stackrel{m}{\sim}, \underset{\sim}{\psi}$ satisfy

$$
\begin{aligned}
& \int_{\Omega} \mathbf{B} \underset{\approx}{\rho_{h}^{m}}: \underset{\approx}{\tau} d x-\sum_{T \in \tau_{h}} \int_{T} \underset{\approx}{\operatorname{grad}} \underset{\sim}{\psi_{h}}: \underset{\approx}{\tau} d x=0 \quad \forall \underset{\approx}{\sim} \in \underset{\approx}{R_{h}^{-1}}, \\
& \sum_{T \in \tau_{h}} \int_{T}{\underset{\approx}{\sim}}_{h}^{m}: \underset{\approx}{\operatorname{grad}} \underset{\sim}{\chi} d x=-\int_{\Omega} \mathcal{P}_{0} \underset{\sim}{f} \underset{\sim}{v} d x \quad \forall \underset{\sim}{v} \in \underset{\sim}{V_{h}^{0} \cup \underset{\sim}{B^{3}}\left(\tau_{h}\right),}
\end{aligned}
$$

where $\mathcal{P}_{0} \underset{\sim}{f}$ denotes the $L^{2}$ projection of $\underset{\sim}{f}$ into piecewise constants.

We next observe that $\underset{\approx}{R_{h}^{-1}}=\underset{\approx}{H_{h}}+\underset{\approx}{L_{h}}$, where $\underset{\approx}{L_{h}}$ is the set of $\underset{\approx}{\tau}$ which on each triangle $T$ have the form

$$
\underset{\approx}{\tau}=\left(\begin{array}{ll}
b_{1}(x-\bar{x}) & b_{1}(y-\bar{y}) \\
b_{2}(x-\bar{x}) & b_{2}(y-\bar{y})
\end{array}\right)
$$

where $b_{1}$ and $b_{2}$ are constants and $\bar{x}$ and $\bar{y}$ denote the average values on $T$ of $x$ and $y$, respectively. We note further that this decomposition is orthogonal in $\underset{\approx}{L^{2}}(\Omega)$. Hence, we may write

$$
\rho_{h}^{m}={\underset{\approx}{\rho}}_{h}^{C}+{\underset{\approx}{\rho}}_{h}^{L}, \quad{\underset{\sim}{\psi}}_{h}={\underset{\sim}{\psi}}_{h}^{L}+{\underset{\sim}{\psi}}_{h}^{B},
$$

with $\underset{\sim}{\rho_{h}^{C}} \in \underset{\approx}{H_{h}}, \underset{\sim}{\rho_{h}^{L}} \in \underset{\approx}{L_{h}}, \underset{\sim}{\psi_{h}^{L}} \in \underset{\sim}{V_{h}^{0}}$, and $\underset{\sim}{\psi_{h}^{B}} \in \underset{\sim}{B^{3}}\left(\tau_{h}\right)$. Since for $\underset{\sim}{\chi} \in \underset{\sim}{V_{h}^{0}}, \underset{\approx}{\operatorname{grad}} \underset{\sim}{\underset{\sim}{\chi}} \underset{\approx}{H_{h}}$ and $\sum_{T \in \tau_{h}} \int_{T} \underset{\approx}{\operatorname{grad}} \underset{\sim}{\chi}: \underset{\approx}{\tau} d x=0$ for $\underset{\sim}{\chi} \in \underset{\sim}{B^{3}}\left(\tau_{h}\right)$ and $\underset{\approx}{\tau} \in \underset{\approx}{H} h$, it is easy to check that $\underset{\sim}{\rho} \rho_{h}^{C}, \underset{\sim}{\psi_{h}^{L}}$ satisfy $(6.5),(6.6)$, with $\underset{\sim}{f}$ replaced by $\mathcal{P}_{0} \underset{\sim}{f}$. If we define $p_{h}^{m}=\operatorname{tr}\left(\underset{\approx}{\rho_{h}^{C}}\right) /(2 \nu-3)$, then by our previous analysis, $\underset{\sim}{\psi}, L, p_{h}^{m}$ satisfy $(6.1),(6.2)$ and ${\underset{\sim}{h}}_{h}^{L}$ satisfies (6.3) with $\underset{\sim}{f}$ again replaced by $\mathcal{P}_{0} \underset{\sim}{f}$. This is exactly analogous to the result derived in [1] for the scalar case. The computations of $\underset{\approx}{\rho} h$ and $\underset{\sim}{\psi_{h}^{B}}$ may then be carried out independently, although the system is somewhat more complicated than the scalar case, due to the presence of the $\operatorname{tr}(\underset{\approx}{\rho}) \underset{\approx}{\delta}$ term. 


\section{REFERENCES}

[1] D. N. ARNold AND F. BREzZI, Mixed and nonconforming finite element methods: implementation, postprocessing, and error estimates, RAIRO Modél. Math. Anal. Numér., 19 (1985), pp. 7-32.

[2] D. N. ARnold And R. S. Falk, A new mixed formulation for elasticity, Numer. Math, 53 (1988), pp. 13-30.

[3] M. Crouzeix AND P.-A. RAviART, Conforming and non-conforming finite element methods for solving the stationary Stokes equations, RAIRO Anal. Numér., 7 (1973), pp. 33-76.

[4] B. M. Fraeijs De Veubeke, A Course in Elasticity, Springer-Verlag, Berlin, New York, 1979.

[5] R. S. FALK, Nonconforming finite elements for the equations of elasticity, preprint.

[6] P. LASCAUX AND P. LESAINT, Some nonconforming finite elements for the plate bending problem, RAIRO Anal. Numér, 9 (1975), pp. 9-53.

[7] L.S.D. MORLEY, The triangular equilibrium element in the solution of plate bending problems, Aero. Quart., 19 (1968), pp. 149-169.

[8] R. RANNACHER, Nonconforming finite element methods for eigenvalue problems in linear plate theory, Numer. Math., 33 (1979), pp. 23-42.

[9] On nonconforming and mixed finite elements for plate bending problems: The linear case, RAIRO Anal. Numér., 13 (1979), pp. 369-387.

[10] P. A. Raviart And J. M. Thomas, A mixed finite element method for second order elliptic problems, in Mathematical Aspects of the Finite Element Method, Lecture Notes in Math. 606, Springer-Verlag, Berlin, 1977. 